\title{
A slanted-nanoaperture metal lens: subdiffraction-limited focusing of light in the intermediate field region
}

\author{
Yun Suk Jung ${ }^{1,2}$, Myungji Kim ${ }^{1,3}$, Yu Shi ${ }^{1}$, Yonggang $\mathrm{Xi}^{1,4}$ and Hong Koo Kim ${ }^{1 *}$ (D)
}

\begin{abstract}
Diffraction of light limits the resolution of beam focusing with conventional lenses, as dictated by the Abbe limit, that is, approximately half the wavelength. Numerous techniques have been explored to overcome this limit. One of the most intensively explored approaches is to design a lens that operates in the near-field region, that is, with a focal length on the order of $10 \mathrm{~nm}$, where evanescent fields can carry and project large in-plane wave-vectors (greater than free-space wave-vectors) to a focal plane. From a practical perspective, however, the requirement of such an ultrashort focal length puts too much constraint, since much longer focal length is commonly desired for intermediate or far-field operation. Here we report a method to beat the Abbe limit while operating with focal length greater than wavelength $\lambda$. Our approach is to tailor the radiation patterns of nanoaperture transmission by tilting aperture axes away from the surface of a metal film such that each slanted aperture transmits a highly directed, tilt-oriented beam onto a common focal point carrying maximal in-plane wave-vector components. The proposed nanoaperture array lens was fabricated by forming tilted nanoslits in a Ag, Al, or $\mathrm{Cr}$ film. We demonstrate minimal spot size of $\lambda / 3$ (210-nm or 110-nm full-width half-maximum at $\lambda=633 \mathrm{~nm}$ or $325 \mathrm{~nm}$, respectively) with $1-4 \lambda$ focal length in air, beating the Abbe limit.
\end{abstract}

Keywords: Metal nanolens, Subdiffraction-limited focusing, Abbe limit, Super-lens, Surface plasmons, Vertical dipole, Radiation pattern, Nanoslit transmission

\section{Introduction}

In beam shaping, a minimal spot size is determined by maximal spatial frequencies available on a focal plane. A variety of different approaches have been reported to overcome the diffraction limit in beam focusing [1-21]. They commonly aim to maximize the spatial frequencies on a focal plane, but with a variable degree of success and limitations. A major challenge outstanding in this field is to achieve both subdiffraction-limited resolution and far-field operation. First, in this Letter we briefly review the underlying principles of diffraction-limited focusing and the limitations entailed by presumed

\footnotetext{
*Correspondence: hkk@pitt.edu

${ }^{1}$ Department of Electrical and Computer Engineering and Petersen Institute of NanoScience and Engineering, University of Pittsburgh, Pittsburgh, PA 15261, USA

Full list of author information is available at the end of the article
}

operating conditions, and explore a method to overcome the problem.

In conventional glass lenses a beam focusing function is performed via refractive transmission of light. In a microscopic view each atomic or molecular level scatterer diffracts incident light into spherical wavefronts, generating so-called Huygens wavelets. Since the spacing of these scatterers is much smaller than wavelength, the wavelets constructively interfere only to the forward direction (i.e., zero-order diffraction) and propagate to far-field without incurring any higher-order diffraction effect. As the wave propagates through lens medium, phase accumulation occurs with an amount proportional to lens thickness and refractive index. At the exit surface of a lens, wavelets emerge with a differing phase relationship, and they constructively interfere and propagate to a direction tilt-oriented from the surface normal. 
One important point to note in this wavelet-based depiction of a refraction phenomenon is that the radiation pattern of each scattering element is intrinsically non-uniform in its angular distribution, that is, maximally transmitting to the forward normal direction and much reduced to tilt-oriented directions. This non-uniform angular distribution corresponds to an obliquity (inclination) factor of wavelet propagation [22]. Let's consider a focal plane placed at far-field. The maximal frequency components available on the focal plane should come from the wavelets emanating from the most offaxis points of a lens, that is, the wavelets with the most glancing propagating (tilt-oriented) wave-vectors toward the focal point. Due to this obliquity factor, however, the high frequency components arrive at a focal plane with much lower intensity than those of normal transmission components. As a result, the maximum spatial frequencies available with a conventional lens are significantly smaller than the free-space wave-vector. The purpose of this paper is to show that the maximal frequency components on a far-field focal plane can be significantly increased by manipulating the radiation pattern of nanoaperture transmission in a metal nanolens.

\section{Transmission through a tilted nanoslit aperture}

A metal nanoslit-array lens can be viewed as a discrete version of conventional glass lens [23]: an incident light transmits through each nanoslit and subsequently reradiates into its characteristic radiation pattern; the wavelets then constructively interfere to the zero-order diffraction direction, similar to the case of conventional dielectric interface described above. However, an important difference should be noted here: in metal lenses the radiation pattern of nanoaperture transmission can be easily altered for the benefit of enhancing the glancing-angle propagation components. Manipulating the nanoaperture transmission patterns corresponds to altering the obliquity factor of wavelet propagation in conventional glass lenses.

In metal nanoaperture transmission, the opposing edges/corners of a nanoaperture opening on exit surface constitute a short dipole that reradiates an aperturetransmitted light into free space [23-25]. In conventional nanoapertures formed in a metal film, aperture opening is usually in-plane horizontal, and therefore the dipole axis is also aligned horizontal, being commonly referred to as a horizontal dipole. The far-field transmission pattern of this in-plane nanoaperture can be modeled as a horizontal dipole placed on metal surface. Similarly, a vertical nanoaperture can also be formed in a metal film by tilting the aperture opening to the normal direction and can be modeled as a vertically-oriented short dipole on metal surface [26-29]. As a generalization of this concept we assume a tilt-oriented short dipole on metal surface (Fig. 1a). The resulting radiation pattern at far-field can be described as a superposition of two copropagating waves: a direct propagation from the short dipole and a reflected (therefore, phase-retarded) propagation from metal surface. The angular $(\phi)$ dependence of the radiation pattern, i.e., field intensity distribution, can be expressed as [29]:

$$
\begin{aligned}
&\langle\boldsymbol{S}\rangle \propto \mid\left(1+r_{p}(\varphi) \exp (i \theta(\varphi))\right) \sin \varphi \cos \alpha \\
& \quad+\left.\left(1-r_{p}(\varphi) \exp (i \theta(\varphi))\right) \cos \varphi \sin \alpha\right|^{2}
\end{aligned}
$$

Here $\alpha$ is the tilt angle of dipole axis referring to the substrate normal, and $\theta$ denotes the amount of phase retardation of reflected transmission.

Figure $1 \mathrm{~b}$ shows the radiation patterns of a horizontal dipole $\left(\alpha=90^{\circ}\right)$ calculated for three different cases of metal: $\mathrm{Ag}, \mathrm{Al}$ and $\mathrm{Cr}$. The following dielectric constants are assumed at $633 \mathrm{~nm}$ wavelength: $\varepsilon=-16.1+i 1.1$ for $\mathrm{Ag} ;-56.5+i 21.2$ for $\mathrm{Al} ;-1.2+i 20.8$ for $\mathrm{Cr}$ [30-33]. The three cases show similar radiation patterns: maximally radiating to the normal direction and with angular width of $\sim 80^{\circ}$ full-width half-maximum (FWHM). Figure 1c shows a vertical dipole case $\left(\alpha=0^{\circ}\right)$. Note that the radiation patterns are highly directed: tilt-oriented to $\sim 65^{\circ}$ from the substrate normal and with significantly smaller FWHM $\left(\sim 43^{\circ}\right)$ than the horizontal dipole case. Among the three metals, $\mathrm{Al}$ shows the largest amount of tilt. Figure $1 \mathrm{~d}$ shows the case of a slanted dipole $\left(\alpha=15^{\circ}\right)$. The radiation patterns orient to the same directions as the vertical dipole case, although the intensity dropped a little bit. A slanted dipole is composed of two dipole components: horizontal and vertical dipoles. For relatively small tilt angles $\left(\alpha<\sim 15^{\circ}\right)$ the radiation pattern is found to be mostly governed by the vertical dipole component.

Figure 2a shows a schematic cross-section of a slanted nanoslit simulated by finite-difference time-domain (FDTD) analysis: 60-nm slit width; $200 \mathrm{~nm} \mathrm{Ag} \mathrm{thickness;}$ $15^{\circ}$ tilt angle of slanted step base. Spherical wavefronts emanate from the vertical nanoslit aperture and propagate to far-field with $47^{\circ}$ tilt of peak-intensity orientation (Fig. 2b, c). This tilt angle is somewhat smaller (by $18^{\circ}$ ) than that of a model calculation of a vertical dipole on a flat horizontal surface (Fig. 1c). This discrepancy is ascribed to the fact that the step base itself in the FDTD simulation case is tilt-oriented (rotated) by $15^{\circ}$. The nearfield region $(y<\sim 200 \mathrm{~nm} ; x<0)$ reveals strong presence of surface plasmons (SPs) excited at slit edge.

The exit side of a nano-aperture generates two wave components: (1) surface plasmons (SP), i.e., a surfacebound wave propagating along the metal surface, and (2) free-space propagating waves emanating from a tilted nanoslit as a dipole (DP) radiation. It might be possible 


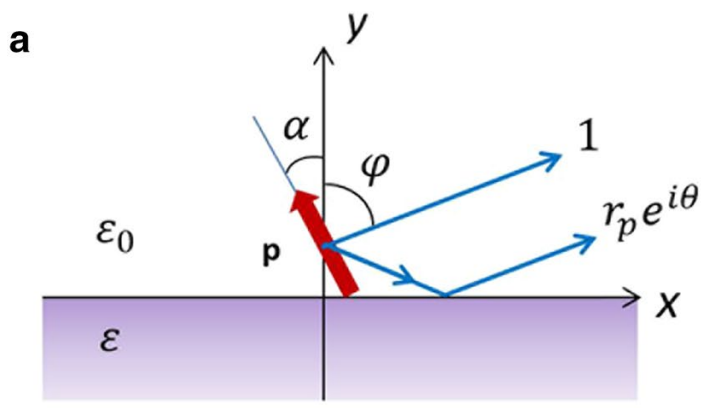

C

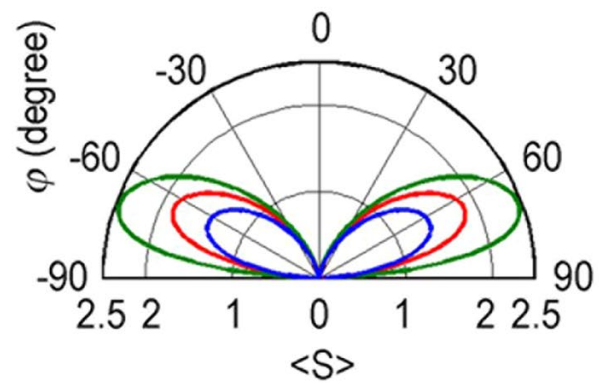

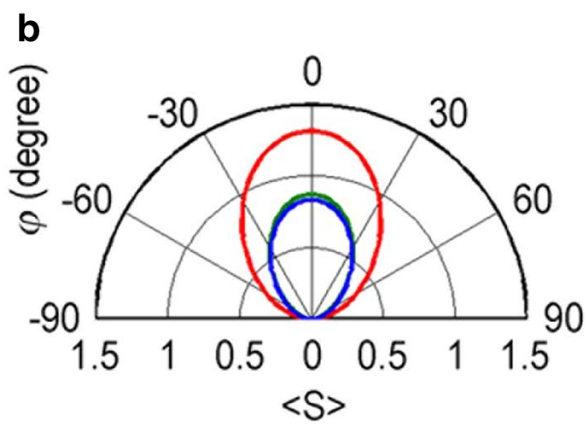

d

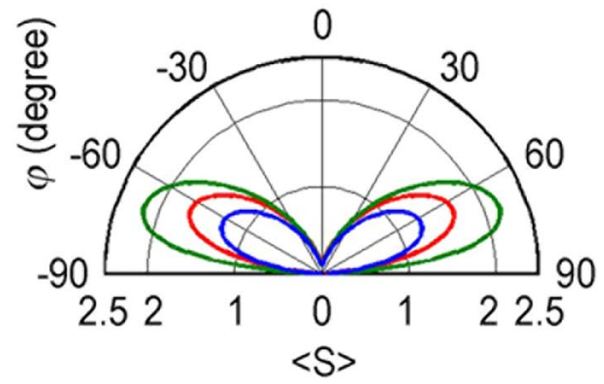

Fig. 1 Dipole radiation patterns. a A tilted dipole placed on metal surface. The tilt angle a refers to the substrate normal. $\mathbf{b}$ Horizontal dipole $\left(a=90^{\circ}\right)$. c Vertical dipole $\left(a=0^{\circ}\right)$. d Titled dipole $\left(a=15^{\circ}\right)$. Three different metals are assumed for substrate: $\mathrm{Ag}$ (red), Al (green) and $\mathrm{Cr}$ (blue). The radiation patterns calculated at $633 \mathrm{~nm}$ wavelength

that the SP fields have reached the focal point with its large wave-vectors and contributed to the sub-diffraction-limited focusing observed in this work. In order to test this possibility we calculated the field distributions of each wave components (SP and DP) and compared their contributions to the focal point. Figure $2 \mathrm{~d}$ shows a field intensity distribution scanned along the normal direction from metal surface to $1 \mu \mathrm{m}$ in air at $x=-2.5 \mu \mathrm{m}$ from a slanted nanoslit. This setting of slit location and observation point corresponds to the same distance between a focal point and the outer-most slit of an 8-slit lens described below (see Fig. 3). Two different field distributions are shown: one from the SP waves (SP, red) and the other one from the radiating components of aperture transmission (DP, blue). Here the total field distribution (SP + DP, black) is decomposed into two parts by taking the following steps: first, in the near-field region we assumed the dominance of SP component over the DP, and read the SP amplitude from the total field plot at metal surface $(y=0)$. The SP penetration depth was then read from a log-linear plot of the total field in the near-field region. The thus-extracted penetration depth is found to well match a theoretical calculation, that is, $390 \mathrm{~nm}$ for Ag/air interface. The DP field distribution is then calculated by subtracting the SP distribution from the total field.
Figure $2 \mathrm{~d}$ reveals that the SP component is dominant only in the near-field region; a cross-over occurs at $y=\sim 0.5 \mu \mathrm{m}$ from the lens surface. At $y=1 \mu \mathrm{m}$, which corresponds to the focal point of an 8-slit lens (see Fig. 3b), the dipole radiation (DP) becomes 10 times stronger than the SP component (Fig. 2d). This confirms that the beam focusing at this focal point $(y=1 \mu \mathrm{m})$ is mostly contributed by the free-space propagating dipole radiation. (In Fig. 3b, the intense short fringes on metal surface are due to interference of counter-propagating SP waves. The evanescent nature of SP fields is evident from the observation that the fringe intensity sharply decays away from the surface with $\sim 0.4 \mu \mathrm{m}$ penetration depth and does not extend to the focal point at $y=1 \mu \mathrm{m}$.)

The tilt-oriented radiation pattern was experimentally demonstrated by employing a slanted nanoslit sample (Fig. 2e), which was fabricated in the following steps. First, a quartz substrate was focused ion-beam (FIB) etched to form a vertical step structure: 200-nm step height and 760-nm lateral span of a tilt-etched step base; this corresponds to $15^{\circ}$ tilt angle of step base. A Ga ion beam was used with dwell time progressively increased for deeper etching along the tilt direction [26]. A 100- to 140-nm-thick Cr film was then deposited on the tilt-etched quartz substrate by thermal evaporation. The resulting slit width on the vertical 
a

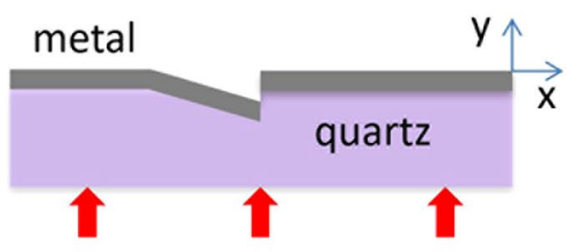

C

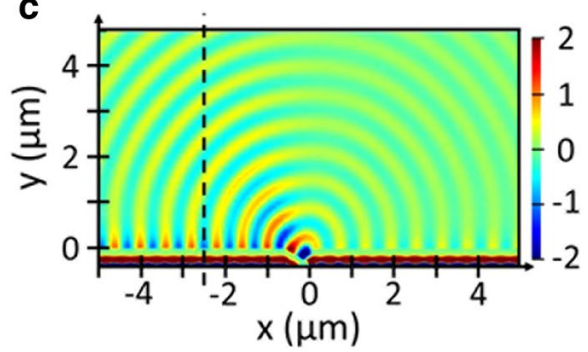

e

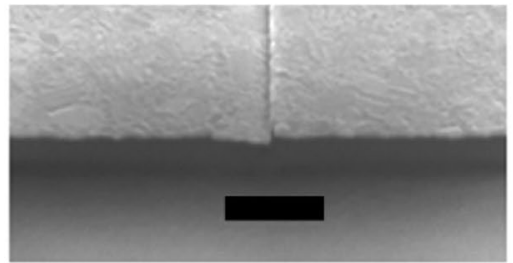

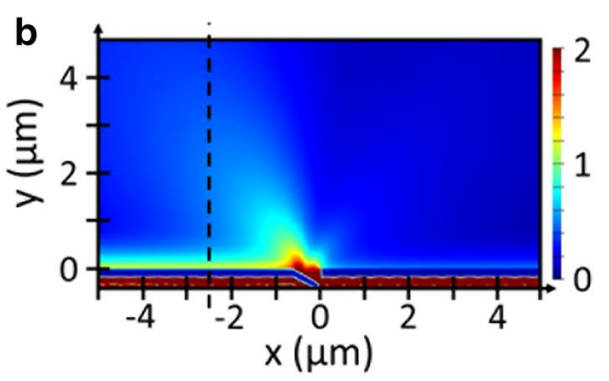

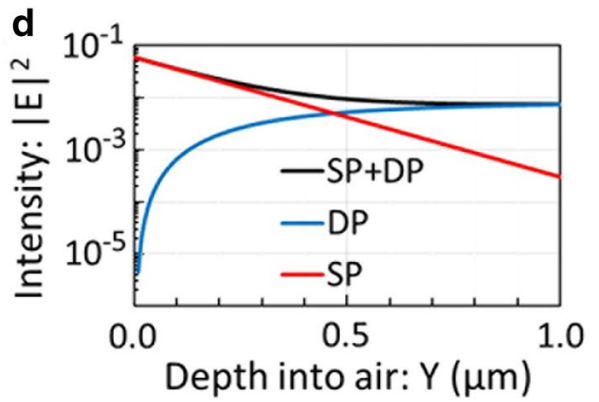

f

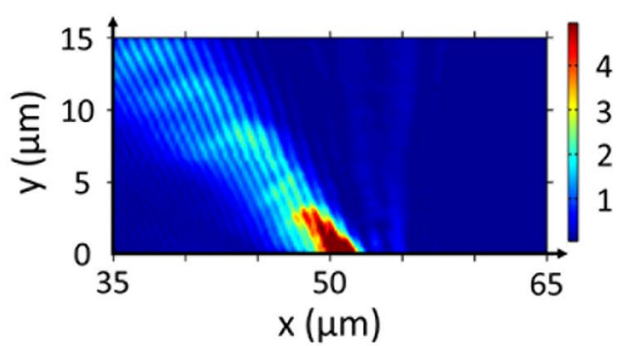

Fig. 2 Transmission through a tilted nanoaperture. a Schematic cross-section of a tilted nanoslit formed in a metal film. A 633-nm TM-polarized light is incident from the bottom side. The transmission patterns calculated by FDTD simulation: $\mathbf{b}$ the distribution of magnetic field amplitude $\left|H_{z}\right| ; \mathbf{c}$ a snapshot image of magnetic field $H_{z}$. Circular wavefronts emanate from the nanoslit aperture with tilted peak-intensity orientation. $\mathbf{d}$ The field intensity distribution scanned along the normal direction at $x=-2.5 \mu \mathrm{m}$ (dashed). The total field (black) is decomposed into two parts: surface-plasmon field (SP, red) and free-space propagating component (DP, blue). e SEM image of a tilted nanoslit aperture formed in a Cr film (100- to $140-n m$ thick). Scale bar, 1 mm. f A beam profile measured by scanning an NSOM probe

sidewall is estimated to be $60-100 \mathrm{~nm}$. Figure $2 \mathrm{f}$ shows the transmission pattern characterized by scanning an apertured near-field scanning optical microscope (NSOM) probe (Veeco Aurora 1720-00: 100-nm-thick Al coated; 80-nm aperture diameter). A TM-polarized light (633 $\mathrm{nm}$ wavelength, $1-\mathrm{mm}$ beam diameter) was incident from the bottom side, and an NSOM probe was scanned on the exit side of the nanoslit aperture. The scan range was $60 \mu \mathrm{m}$ on the horizontal direction and $40 \mu \mathrm{m}$ in the vertical direction. The step size of scan was $50 \mathrm{~nm}$ and $157 \mathrm{~nm}$ in the horizontal and vertical directions, respectively. The NSOM-scan result shows $45^{\circ}$ tilt of the main lobe (Fig. 2f) and compares well with the FDTD of Ag nanoslit (Fig. 2b, c).

\section{Beam focusing with slanted-nanoslit array lenses}

Based on this single-slit result an array of slanted nanoslits is designed for a nanolens that can operate at intermedi-

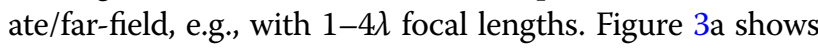
a schematic cross-section of an 8-slit nanolens with focal length of $2 \lambda$ at $633 \mathrm{~nm}$ wavelength in air ambient. The aperture location and orientation of each nanoslit is designed such that their radiation patterns orient to a common focal point and the wavefronts emanating from each slit arrive at the focal point in phase, i.e., constructively interfere there. The thus determined slit locations are: $x= \pm 0.26 \mu \mathrm{m}$ $; \pm 1.26 \mu \mathrm{m} ; \pm 2.04 \mu \mathrm{m} ; \pm 2.78 \mu \mathrm{m}$. Here we also note that the location of the inner-most slit pair (at $x= \pm 0.26 \mu \mathrm{m})$ 
a
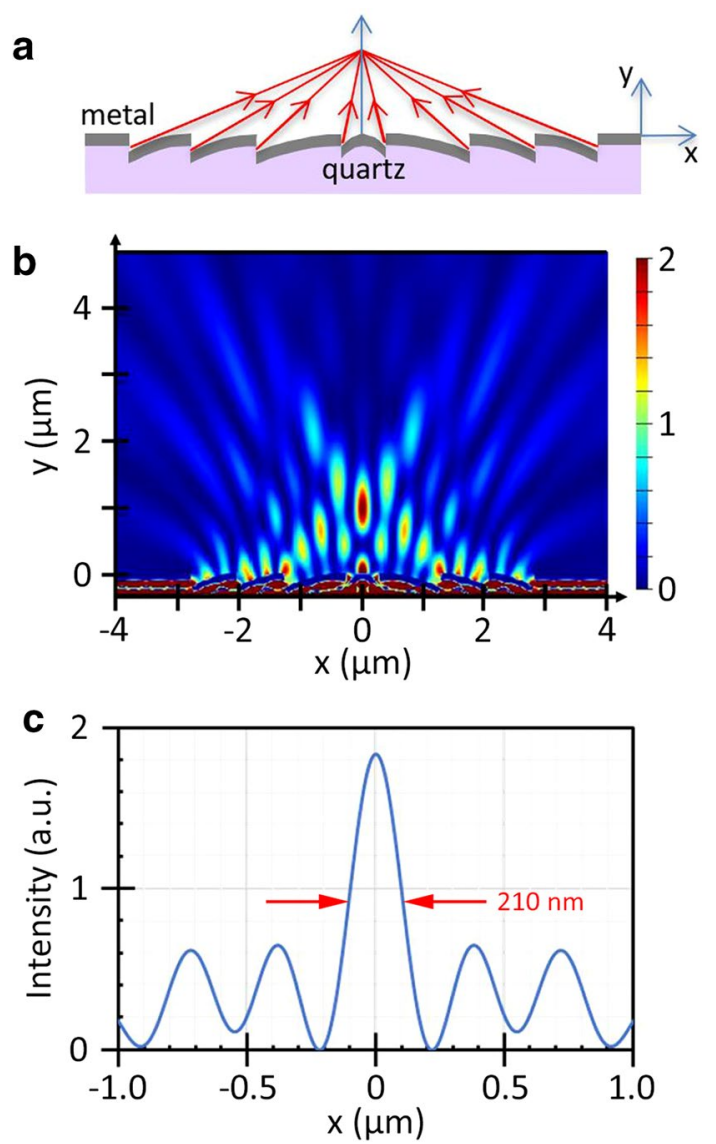

Fig. 3 Beam focusing by a tilted nanoslit array lens. a Schematic cross-section of an 8-slit Ag nanolens designed for $2 \lambda$ focal length at $\lambda=633 \mathrm{~nm}$. b FDTD simulation of transmission pattern: field intensity distribution. $\mathbf{c}$ The intensity distribution scanned at $y=1.0 \mu \mathrm{m}$. The central peak shows $210 \mathrm{~nm}$ FWHM, corresponding to $0.33 \lambda$

is determined taking into account the phase-retardation effect of SPs' lateral propagation to the opposing slit, where they decouple into free space radiation.

Figure $3 \mathrm{~b}$ shows FDTD simulation of transmission through the 8-nanoslit array Ag lens. The scan profile at the focal plane $(y=1.0 \mu \mathrm{m})$ is also shown. The central peak accounts for $30 \%$ of total beam power crossing the plane. The FWHM of this main peak is measured to be $210 \mathrm{~nm}$. This corresponds to $0.33 \lambda$, smaller than the Abbe limit $(\sim \lambda / 2)$. Each nanoslit pair generates two propagating waves with the following in-plane wave-vectors when projected to a focal plane: $\pm k_{0} \sin \theta$. The resulting interference pattern can be expressed as:

$$
\left|e^{i\left(k_{0} \sin \theta\right) x}+e^{-i\left(k_{0} \sin \theta\right) x}\right|^{2} \propto \cos ^{2}\left(\left(k_{0} \sin \theta\right) x\right) .
$$

For each slit pair the corresponding fringe width $\left(\pi / 2 k_{0} \sin \theta ; \mathrm{FWHM}\right)$ is calculated to be $167 \mathrm{~nm}, 175 \mathrm{~nm}$,
$199 \mathrm{~nm}$, and $607 \mathrm{~nm}$, counting from the outer-most slit to the inner ones. The resulting beam profile of the 8 -slit nanolens is found to broaden a little bit (to $210 \mathrm{~nm}$ FWHM of the central peak: see Fig. 3c), but this width is still significantly smaller than the Abbe limit $(\sim 316 \mathrm{~nm})$ at $633 \mathrm{~nm}$ wavelength.

Accurately measuring the minimal spot size at a given focal plane is crucially important in our efforts to draw a solid, unambiguous conclusion on whether we beat the Abbe limit or not. The accuracy of our NSOM measurement was tested with reference samples that were fabricated for this study. A single horizontal nanoslit structure was fabricated by FIB etching of a Cr film (65-nm thick) deposited on quartz substrate, with three different slit widths: $60 \mathrm{~nm}, 120 \mathrm{~nm}$ and $180 \mathrm{~nm}$; see Fig. 4a-c, respectively. Figure 4 (bottom panel) shows NSOM-scan profiles measured at three different heights $(y=0.16 \mu \mathrm{m}$, $0.31 \mu \mathrm{m}$, and $0.47 \mu \mathrm{m})$ at $633 \mathrm{~nm}$ wavelength: the step size of horizontal scan was $10 \mathrm{~nm}$. The three scan profiles show the same FWHM values for each slit: $92 \mathrm{~nm}$ for 60-nm slit, $120 \mathrm{~nm}$ for 120 -nm slit, and $178 \mathrm{~nm}$ for 180 $\mathrm{nm}$ slit. Note the excellent agreement between NSOM and SEM measurements for the 120-nm and 180-nm slit samples and the large discrepancy ( $92 \mathrm{~nm}$ by NSOM versus $60 \mathrm{~nm}$ by SEM) for the $60-\mathrm{nm}$ slit sample case. This measurement result suggests that the resolution of our NSOM scan is better (smaller) than $120 \mathrm{~nm}$.

The designed nanoslit array lens structure was fabricated on quartz substrate by FIB etching and followed by metal deposition: Fig. 5a for SEM images (top: side view; bottom: plan view). Note the symmetric configuration of aperture orientations across the lens axis. After FIB etching of four tilted-steps on one side, the same FIB process was repeated to complete the other four steps with an opposite tilt orientation. The overall dimensions of an 8-slit array lens are: $5.6-\mu \mathrm{m}$ width (lateral span of 8-slit array) and $50-\mu \mathrm{m}$ length (slit length). An $\mathrm{Ag}(150 \mathrm{~nm}), \mathrm{Al}$ $(150 \mathrm{~nm})$ or $\mathrm{Cr}(80 \mathrm{~nm})$ film was then deposited by thermal evaporation with an incident flux normal to the substrate. The resulting slit width on the vertical sidewall is estimated to be $60-100 \mathrm{~nm}$.

The beam focusing function of the fabricated nanolenses was characterized by NSOM technique. A TMpolarized light $(633 \mathrm{~nm}$ or $325 \mathrm{~nm}$ wavelength; $1 \mathrm{~mm}$ beam diameter) was incident from the bottom side, and an NSOM probe was scanned in the horizontal direction in the following window: $-10 \mu \mathrm{m}<x<+10 \mu \mathrm{m}$; $0 \mu \mathrm{m}<y<7 \mu \mathrm{m}$. The scan step size was $10 \mathrm{~nm}$ for $x$-direction and $157 \mathrm{~nm}$ for $y$-direction. For the high resolution (10 $\mathrm{nm}$ step) scan in the $x$-direction, a piezo servo-controller (PI E-665.CR) and a nano-stage (PI P-621.1CD) were used with 5 -ms integration time, $150-\mu$ s dead time and 200-ms step delay. For the $y$-axis scan, a piezo driver 

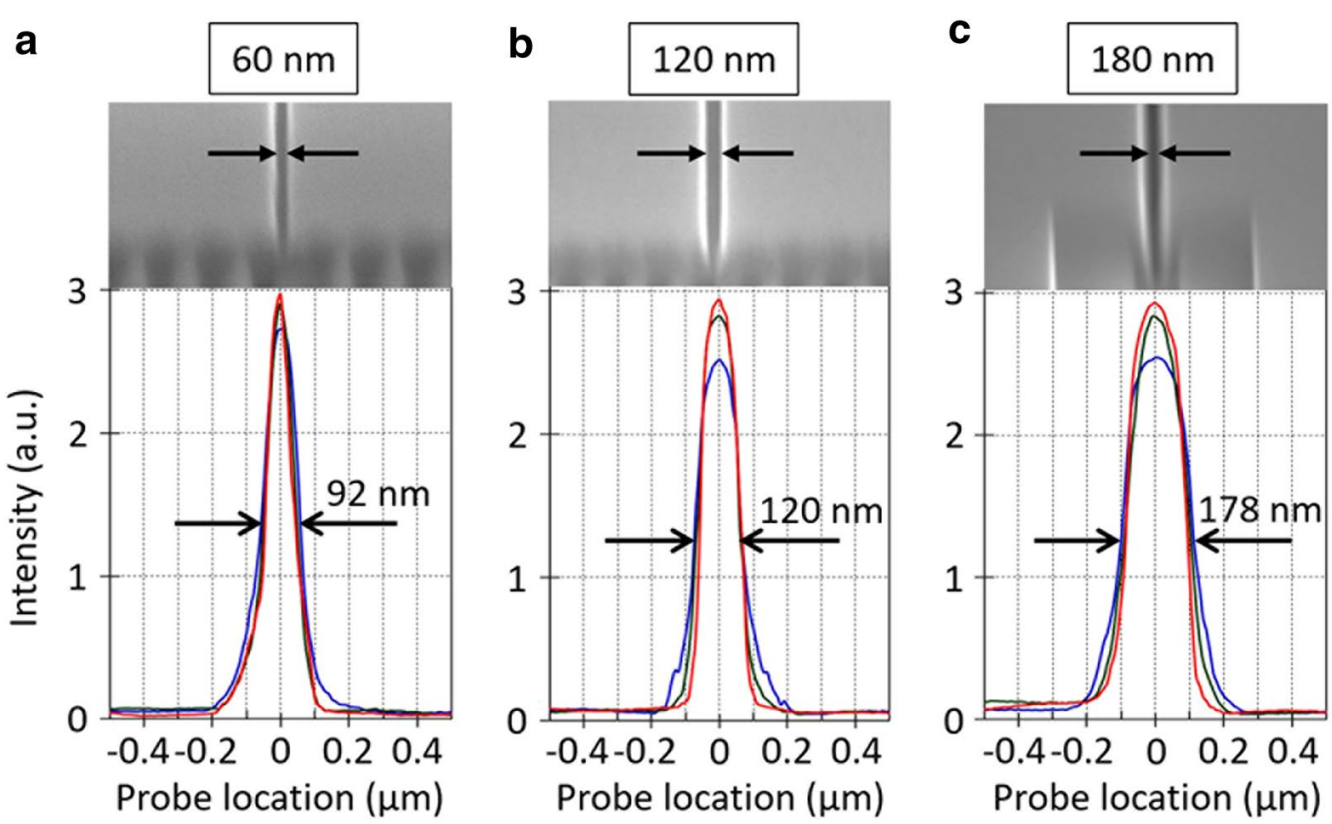

Fig. 4 Spatial resolution of NSOM scanning. A single horizontal nanoslit (slit width of $60 \mathrm{~nm}, 120 \mathrm{~nm}$, or $180 \mathrm{~nm}$ ) was formed in a Cr film (65-nm thick): see top panels for SEM images. The NSOM scan profiles at $y=0.16 \mu \mathrm{m}$ (red), $0.31 \mu \mathrm{m}$ (green), and $0.47 \mu \mathrm{m}$ (blue) at $633 \mathrm{~nm}$ wavelength: see bottom panels. a 60-nm slit sample showing $92 \mathrm{~nm}$ FWHM. b 120-nm slit sample showing $120 \mathrm{~nm}$ FWHM. c 180-nm slit sample showing $178 \mathrm{~nm}$ FWHM
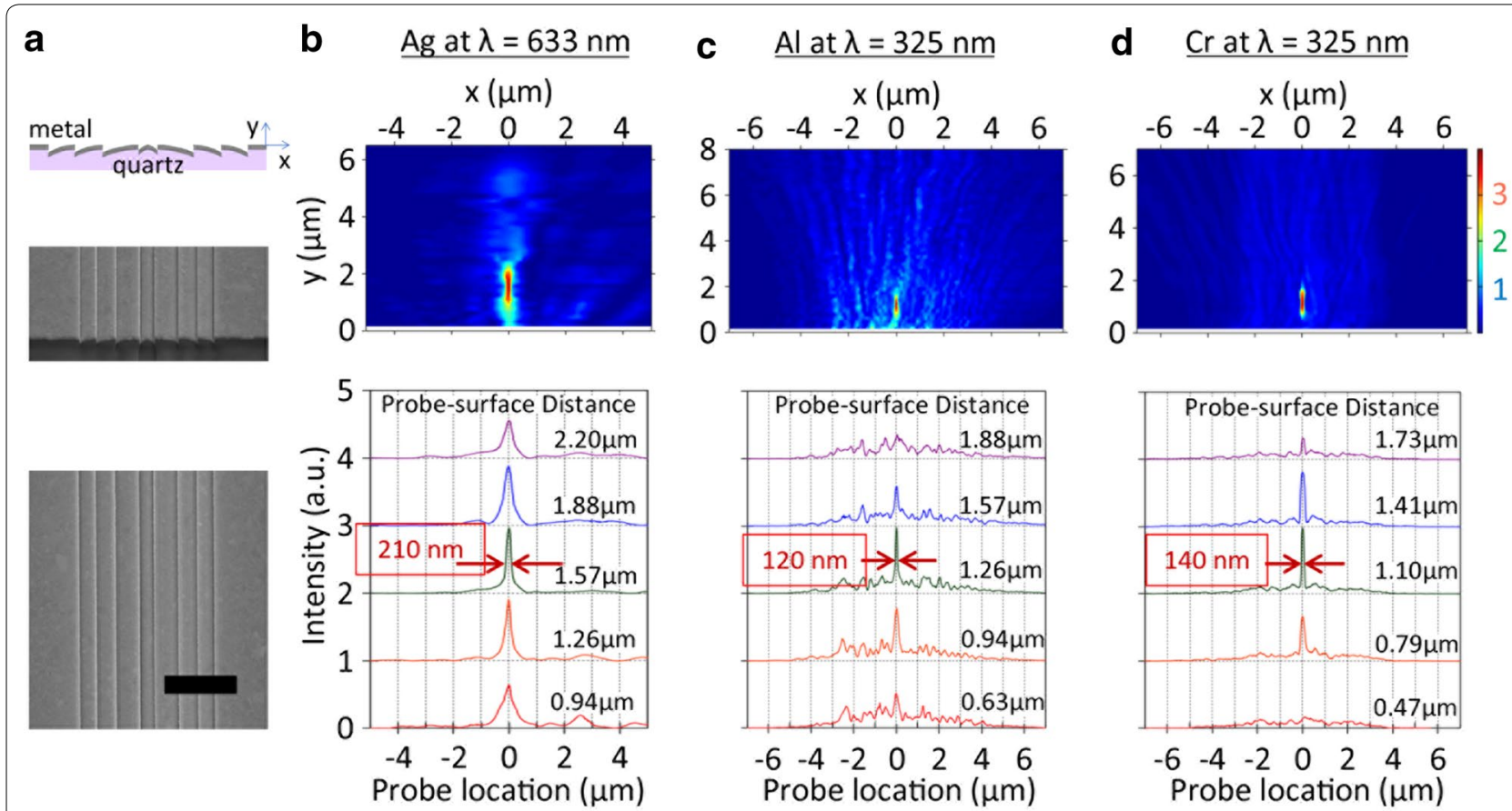

Fig. 5 Subdiffraction-limited focusing of light with a tilted nanoslit array lens. a SEM image of an 8-slit Ag lens. Scale bar, $3 \mu \mathrm{m}$. b Ag lens characterized at $633 \mathrm{~nm}$ wavelength. NSOM scan image (top) and scan profiles (bottom). A minimal spot size is measured to be $210 \mathrm{~nm}$ FWHM at $y=1.57 \mu \mathrm{m}$. c Al lens at $325 \mathrm{~nm}$ wavelength. A minimal spot size of $120 \mathrm{~nm}$ is observed at $y=1.26 \mu \mathrm{m}$. $\mathbf{d}$ Cr lens at $325 \mathrm{~nm}$ wavelength. A minimal spot size of $140 \mathrm{~nm}$ is observed at $y=1.10 \mu \mathrm{m}$ 
and stage (Melles-Griot 17 PCS 001 and NanoBlock 3-axes Flexure Stage) were used with a 157-nm step size. An aperture-NSOM probe was initially brought to the proximity of a sample; the probe was further moved forward touching the sample surface. After this zero-point checking the probe was retracted back by one step (157$n m$ step size) and a scanning ensued. This NSOM scanning experiment was performed on a vibration isolation table.

Figure $5 \mathrm{~b}$ shows the result with an 8-nanoslit Ag lens measured at $633 \mathrm{~nm}$ wavelength. Scan profiles are shown at several probe-surface distances (bottom panel). In the intermediate field region $(y-\lambda)$ the beam profile becomes simpler and narrower. A minimal spot size (FWHM) is measured to be $210 \mathrm{~nm}$ at $y=1.57 \mu \mathrm{m}$. This corresponds to $\lambda / 3$ FWHM, smaller than the Abbe limit.

Figure $5 \mathrm{c}, \mathrm{d}$ shows the NSOM-scanned transmission patterns of 8-slit $\mathrm{Al}$ or $\mathrm{Cr}$ lenses designed for $4 \lambda$ focal length at $325 \mathrm{~nm}$ wavelength. A focal plane is observed at $1.26 \mu \mathrm{m}$ (for $\mathrm{Al}$ ) or $1.10 \mu \mathrm{m}$ distance (for $\mathrm{Cr}$ ) from the lens surface, corresponding to $3.9 \lambda$ or $3.4 \lambda$ focal length, respectively. The minimal spot size (FWHM) is measured to be $120 \mathrm{~nm}$ (for $\mathrm{Al}$ ) or $140 \mathrm{~nm}$ (for Cr). These FWHM values correspond to $0.37 \lambda$ or $0.43 \lambda$, again smaller than the Abbe limit. It is interesting to note that the minimal spot size of the $\mathrm{Al}$ lens is slightly smaller than that of the $\mathrm{Cr}$ lens. This is ascribed to the fact that the radiation pattern of a vertical dipole on $\mathrm{Al}$ surface is more tilt-oriented (not shown in this paper) and therefore providing larger in-plane wave-vectors than the $\mathrm{Cr}$ case.

The dominance of the aperture radiation contribution over SP's to sub-diffraction-limited focusing is confirmed in this experiment with a $\mathrm{Cr}$ lens characterized at $325 \mathrm{~nm}$ wavelength (Fig. 5d). The Cr/air interface poorly supports SPs at this wavelength: the propagation length and the penetration depth into air are calculated to be $0.5 \mu \mathrm{m}$ and $190 \mathrm{~nm}$, respectively, assuming $\varepsilon=-3.5+i 8.4$ [31-33]. With such a small penetration depth and propagation length, a SP contribution to the focal point at $y=1 \mu \mathrm{m}$ is expected to be negligible, and yet the $\mathrm{Cr}$ lens demonstrates sub-Abbe-limit focusing (140 nm FWHM $<163 \mathrm{~nm}$ of Abbe limit). This supports our conclusion on DP fields' dominant contribution to the subdiffraction-limited focusing at far-field of our lens.

We also calculated the transmitted power of a horizontal nanoslit and a slanted nanoslit formed in a 150-nmthick film with the same amount of aperture opening (slit width of $60 \mathrm{~nm}$ ). At $633 \mathrm{~nm}$ wavelength, the total transmitted power of the slanted nanoslit is found to be $\sim 45 \%$ of the horizontal nanoslit's. This difference is partly ascribed to a smaller effective-aperture-area of a slanted nanoslit seen by an incident beam. The transmission efficiency of the horizontal nanoslit is estimated to be $120 \%$.
Here the transmission efficiency is defined as the ratio of total transmitted power to the incident power that falls on the aperture area, that is, the throughput power is normalized by the aperture area seen from the incident beam direction. It is interesting to note that the transmission efficiency is greater than $100 \%$. This implies the nanoslit transmission involves a funneling effect occurring at aperture edges on the incident side [34]. In the case of the 8 slanted nanoslit array lens (Fig. 3a), the transmission efficiency is estimated to be $4.8 \%$. In this estimation, the total transmitted power is normalized by the input power that falls on the entire slit array, that is, $5.6 \mu \mathrm{m}$ wide area. Considering the relatively large spacing between nanoslits, the reduction of overall transmission is explained by the fact that the total, effective aperture areas of 8 slits account for a small portion of the total lens area.

\section{Comparison with super-oscillatory lenses}

It is highly instructive to compare the results and mechanisms of this work with those of another interesting technique that also demonstrates subdiffraction-limited focusing at far-field. It is well known in literature that a band-limited function can oscillate faster than their fastest Fourier components, generating so-called superoscillations $[35,36]$. According to this theory, an arbitrarily small (i.e., narrow) focal spot can form at far-field of a properly-designed super-oscillatory lens, which looks to violate the Fourier transform relationship. A recent experimental work also demonstrates $0.45 \lambda$ FWHM with $25 \lambda$ focal length by employing a nanoaperture-array metal lens designed by this super-oscillation principle [37]. In fact, this seemingly paradoxical behavior does not contradict the Fourier relationship and can be explained as follows.

First, in Fourier theory the spatial frequency components refer to the entire beam profile defined in a global perspective, whereas in the super-oscillation theory they are locally defined in a narrow region. In this local frequency concept, presence of a narrow peak implies involvement of local large wave-vectors there, implying a rapid change of optical phase in that region. The local wave-vectors in this super-oscillatory region commonly exceed 10 times the free-space wave-vector [36]. This super-oscillation is an interference effect of band-limited propagating waves and requires a precise and delicate control of both amplitude and phase of Fourier components. It is important to note that a superposition of these propagating waves results in high-intensity side lobes that surround the narrow peak of much lower intensity in the central region. There also exists a tradeoff between the narrow peak and side lobes: the narrower the central peak the stronger the intensity of side lobes. As the 
central peak becomes narrower, the sidebands shift closer to the center, making it difficult to separate them. For example, the intensity of a central peak with $\lambda / 5$ FWHM is $10^{-7}$ times weaker than that of sidebands, and the peak is separated from sidebands by $0.5 \lambda$ distance [36].

By contrast, our approach is to manipulate the angular distribution of aperture radiation by tilting the aperture axis such that the transmitted power is directed into a focal spot at far-field with a reduced amount of solid angle. The directed beaming nature of the slanted nanoslit is the core difference with the conventional nanoslit structure case, in which a horizontally-open nanoslit radiates into the normal direction with larger solid-angle. In our nanoslit-array lens the aperture transmission from the outermost slit is the most glancingangle propagating, and therefore it carries the largest wave-vector component to a focal plane. It should be noted that the aperture transmissions emerging from the slits are all in-phase. In general, this phase relationship can be included as a design parameter in a way similar to designing a super-oscillation lens [36]. In the current work, this phase factor was not taken into further optimization. Instead, by manipulating the obliquity factor

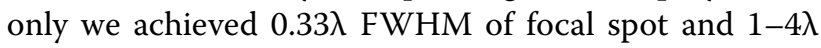
focal length. This focal spot accounts for $30 \%$ of total transmitted power, and no major side lobes appear on the focal plane. To the authors' knowledge, this spot width is

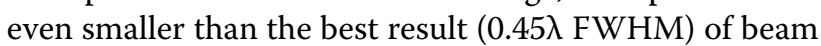
focusing with a super-oscillation lens reported in the literature [37].

Note that the focal length of our lens scales up with the lateral dimension of a lens, that is, the size of nanoslit array, and the maximum glancing angle of aperture transmission. In the present work we obtained $4 \lambda$ focal length from a 5.6- $\mu \mathrm{m}$ size array lens. By employing a larger size lens the focal length can be further increased, for example, $40 \lambda$ focal length from a $56-\mu \mathrm{m}$ lens while maintaining the same level of sub-Abbe-limit focusing. This scalability can be compared with the super-oscillatory lens that demonstrates $25 \lambda$ focal length from a $40-\mu \mathrm{m}$ diameter lens [37]. It should also be noted that the focal spot size can be proportionally scaled down to much smaller width by employing a high-index immersion optics configuration. Ref [38] reports an experimental work that demonstrates sub-50 nm FWHM focal spot of a superoscillatory lens placed in high-index solid-state immersion ambient (GaP with a refractive index of 3.72).

Table 1 summarizes comparisons of the three different nanolenses that demonstrate sub-Abbe-limited beam focusing.

\section{Beam focusing effects of an all-dielectric slanted-nanoslit array lens}

As an alternative to metallic metamaterials, all-dielectric metamaterials hold promising potential for nano-optic applications, where energy loss is an important factor that would limit device performance [39]. In the present work we also simulated beam-focusing effects of an all-dielectric slanted-slit array nanolens that employs $\mathrm{Si}$ instead of Ag for nano-aperture formation. Figure 6 shows a FDTD analysis of beam focusing effect of a dielectric nanolens at $633 \mathrm{~nm}$ wavelength. Here we assumed the same structure and dimensions as the 8-slit Ag lens discussed above (Fig. 5a): a Ag layer (150 nm thick) was simply replaced by a Si layer of the same thickness. Figure 6a shows an overall transmission pattern, and Fig. $6 \mathrm{~b}$ an intensity profile scanned at the focal plane $(y=1.5 \mu \mathrm{m})$. Interesting observations can be made as follows. First, the FWHM of the center peak is measured to be $260 \mathrm{~nm}$, corresponding to $0.41 \lambda$. This width is somewhat larger than the $\mathrm{Ag}$ case $(210 \mathrm{~nm})$, but still smaller than the Abbe limit. The entire region is crowded with many interference fringes of high intensity. In the scan profile, these fringes appear as intense side-lobes and they account for a major portion of the total transmitted power.

The many-fringe nature of this $\mathrm{Si}$ lens's transmission pattern is explained as follows. First, two types of waves co-exist in the transmission side: direct transmission through a Si layer and aperture transmission through 8 tilted-nanoslits formed in $\mathrm{Si}$. Two different types of interference patterns will then be generated: (1) between direct transmission and aperture transmission and (2) between two aperture transmissions coming from opposing directions. Type 1 interference involves

Table 1 Comparison of a slanted nanoslit array lens with superoscillatory lenses: sub-Abbe-limit beam focusing at farfield

\begin{tabular}{|c|c|c|c|c|c|c|c|}
\hline Type of lens and ambient & Materials & $\begin{array}{l}\text { Size } \\
(\mu \mathrm{m})\end{array}$ & $\begin{array}{l}\text { Working } \\
\text { wavelength, } \lambda \\
(\mathrm{nm})\end{array}$ & Spot size, FWHM & Focal length & Power ratio & References \\
\hline Slanted nanoslit array in air & Air/Ag/glass & 5.6 & $\begin{array}{l}633 \\
325\end{array}$ & $\begin{array}{l}210 \mathrm{~nm}(0.33 \lambda) \\
120 \mathrm{~nm}(0.37 \lambda)\end{array}$ & $\begin{array}{l}1.6 \mu \mathrm{m}(2.5 \lambda) \\
1.3 \mu \mathrm{m}(4 \lambda)\end{array}$ & $4.8 \%$ & This work \\
\hline Super-oscillatory in air & Air/Au/glass & 40 & 405 & $185 \mathrm{~nm}(0.45 \lambda)$ & $10 \mu \mathrm{m}(25 \lambda)$ & - & {$[37]$} \\
\hline Super-oscillatory in GaP $(n=3.72)$ & GaP/metal/glass & 42 & 473 & $57 \mathrm{~nm}(0.12 \lambda)$ & $5 \mu \mathrm{m}(11 \lambda)$ & $1.8 \%$ & [38] \\
\hline
\end{tabular}



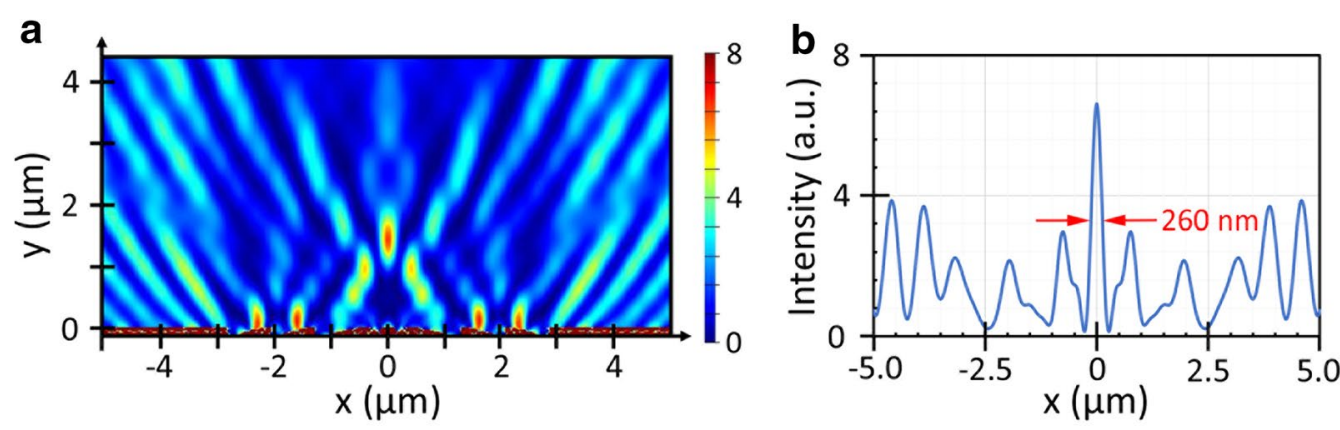

Fig. 6 Beam focusing effects of an all-dielectric (Si/silica) slanted-nanoslit-array nanolens. a FDTD simulation of transmission pattern simulated at $633 \mathrm{~nm}$ wavelength. The same structure and dimensions are assumed as the Ag lens case (Fig. 3). b The intensity profile scanned at the focal plane

planar wavefronts (of direct transmission) and circular wavefronts (of aperture transmission). The resulting fringe pattern evolves into a parabolic profile at far-field [40]. Type 2 interference involves two circular wavefronts arriving from opposing directions, and produces the central peak and satellite peaks at the focal plane. The direct transmission through a $\mathrm{Si}(150 \mathrm{~nm}) / \mathrm{SiO}_{2}$ layer is estimated to be $63 \%$ (transmittance). It is interesting to note that the central peak is of similar intensity with side lobes. This implies that the transmission through a $\mathrm{Si}$ nanoslit aperture is reasonably strong. In brief, the following conclusions can be drawn from this simulation study: a far-field sub-diffraction-limited beam focusing is demonstrated with an all-dielectric $\left(\mathrm{Si} / \mathrm{SiO}_{2}\right)$ nanolens that employs a slanted nanoslit array for glancing angle transmission of light; the direct transmission through the dielectric layers is found to be strong, generating many side lobes of high intensity.

\section{Conclusions}

We have developed a slanted-nanoslit array lens structure and demonstrate a subdiffraction-limited $(\lambda / 3)$ focusing of UV-visible light ( $325 \mathrm{~nm}$ or $633 \mathrm{~nm}$ ) with focal length of $1-4 \lambda$. This sub-Abbe-limited focusing was enabled by manipulating the far-field transmission patterns of nanoslit apertures for more glancing-angle propagation, and thereby increasing the maximal in-plane wave-vectors. This method corresponds to altering the obliquity factor of wavelet propagation and offers a new degree of freedom in designing far-field super-resolution lenses.

\section{Authors' contributions}

YSJ performed sample fabrication and characterization. MK carried out analytical model calculation. YS and YX performed FDTD analysis. HKK conceived and supervised this project. All authors contributed to writing this manuscript. All authors read and approved the final manuscript.

\section{Author details}

${ }^{1}$ Department of Electrical and Computer Engineering and Petersen Institute of NanoScience and Engineering, University of Pittsburgh, Pittsburgh, PA 15261, USA. ${ }^{2}$ Present Address: ALKOR Semi, Cohoes, NY 12047, USA. ${ }^{3}$ Present
Address: Samsung Display, Yongin, South Korea. ${ }^{4}$ Present Address: Google, Venice, CA 90291, USA

\section{Competing interests}

The authors declare that they have no competing interests.

Availability of data and materials

The authors have no data to share since all data are shown in the submitted manuscript.

Ethics approval and consent to participate Not applicable.

\section{Funding}

This work was supported by grants from the NSF (NIRT-ECS-0403865, ECS0424210 and ECCS-0925532).

\section{Publisher's Note}

Springer Nature remains neutral with regard to jurisdictional claims in published maps and institutional affiliations.

Received: 24 September 2018 Accepted: 8 November 2018 Published online: 26 November 2018

\footnotetext{
References

1. V.G. Veselago, Sov. Phys. Uspekhi 10, 509 (1968)

2. S.M. Mansfield, G.S. Kino, Appl. Phys. Lett. 57, 2615 (1990)

3. E. Yablonovitch, R.B. Vrijen, Opt. Eng. 38, 334 (1999)

4. J.B. Pendry, Phys. Rev. Lett. 85, 3966 (2000)

5. A.N. Boto, P. Kok, D.S. Abrams, S.L. Braunstein, C.P. Williams, J.P. Dowling, Phys. Rev. Lett. 85, 2733 (2000)

6. I.A. Larkin, M.I. Stockman, Nano Lett. 5, 339 (2005)

7. N. Fang, H. Lee, C. Sun, X. Zhang, Science 308, 534 (2005)

8. V.A. Podolskiy, E.E. Narimanov, Opt. Lett. 30, 75 (2005)

9. S. Durant, Z.W. Liu, J.A. Steele, X. Zhang, J. Opt. Soc. Am. B 23, 2383 (2006)

10. Z. Jacob, L.V. Alekseyev, E. Narimanov, Opt. Express 14, 8247 (2006)

11. Z.W. Liu, H. Lee, Y. Xiong, C. Sun, X. Zhang, Science 315, 1686 (2007)

12. I.I. Smolyaninov, Y.J. Hung, C.C. Davis, Science 315, 1699 (2007)

13. Y. Fu, W. Zhou, L.E.N. Lim, C.L. Du, X.G. Luo, Appl. Phys. Lett. 91, 061124 (2007)

14. X. Zhang, Z.W. Liu, Nat. Mater. 7, 435 (2008)

15. N.I. Zheludev, Nat. Mater. 7, 420 (2008)

16. F.M. Huang, N.I. Zheludev, Nano Lett. 9, 1249 (2009)

17. S. Gazit, A. Szameit, Y.C. Eldar, M. Segev, Opt. Express 17, 23920 (2009)

18. D. Choi, Y. Lim, S. Roh, I.M. Lee, J. Jung, B. Lee, Appl. Opt. 49, A30 (2010)

19. F. Aieta, P. Genevet, M.A. Kats, N.F. Yu, R. Blanchard, Z. Gahurro, F. Capasso, Nano Lett. 12, 4932 (2012)

20. A.V. Kildishev, A. Boltasseva, V.M. Shalaev, Science 339, 1232009 (2013)
} 
21. E. Centeno, A. Moreau, Phys. Rev. B 92, 045404 (2015)

22. M. Born, E. Wolf, A.B. Bhatia, Principles of Optics: Electromagnetic Theory of Propagation, Interference and Diffraction of Light, 7th (expanded) edn edn. (Cambridge University Press, Cambridge, 1999)

23. Z.J. Sun, H.K. Kim, Appl. Phys. Lett. 85, 642 (2004)

24. W.L. Barnes, A. Dereux, T.W. Ebbesen, Nature 424, 824 (2003)

25. Y. Xie, A.R. Zakharian, J.V. Moloney, M. Mansuripur, Opt. Express 12, 6106 (2004)

26. M. Kim, Y.S. Jung, Y. Xi, H.K. Kim, Appl. Phys. Lett. 107, 101107 (2015)

27. L. Novotny, B. Hecht, Principles of Nano-Optics, 2nd edn. ((Cambridge University Press, Cambridge, 2012)

28. J. Bravo-Abad, L. Martin-Moreno, F.J. Garcia-Vidal, Phys. Rev. E 69, 026601 (2004)

29. G. Brucoli, L. Martin-Moreno, Phys. Rev. B 83, 045422 (2011)
30. E.D. Palik, Handbook of Optical Constants of Solids I (Academic Press, Orlando, 1985)

31. P.B. Johnson, R.W. Christy, Phys. Rev. B 6, 4370 (1972)

32. P.B. Johnson, R.W. Christy, Phys. Rev. B 9, 5056 (1974)

33. A.D. Rakic, Appl. Opt. 34, 4755 (1995)

34. Y.G. Xi, Y.S. Jung, H.K. Kim, Opt. Express 18, 2588 (2010)

35. M.V. Berry, S. Popescu, J. Phys. A 39, 6965 (2006)

36. E.T.F. Rogers, N.I. Zheludev, J Opt. 15, 094008 (2013)

37. G.H. Yuan, E.T.F. Rogers, T. Roy, G. Adamo, Z.X. Shen, N.I. Zheludev, Sci. Rep. 4, 6333 (2014)

38. G.H. Yuan, E.T.F. Rogers, T. Roy, Z.X. Shen, N.I. Zheludev, Opt. Express 22, 6428 (2014)

39. S. Jahani, Z. Jacob, Nat. Nanotechnol. 11, 23 (2016)

40. Y.S. Jung, Y.G. Xi, J. Wuenschell, H.K. Kim, Opt. Express 16, 18881 (2008)

\section{Submit your manuscript to a SpringerOpen ${ }^{\odot}$ journal and benefit from:}

- Convenient online submission

- Rigorous peer review

- Open access: articles freely available online

- High visibility within the field

- Retaining the copyright to your article

Submit your next manuscript at $\boldsymbol{\Delta}$ springeropen.com 